\title{
Scintillation Properties and $\alpha$-ray Detection Capabilities of Thin-film Plastic Scintillators
}

\author{
Masanori Koshimizu, ${ }^{1 *}$ Takayuki Yanagida, ${ }^{2}$ Ryoji Kamishima, ${ }^{3}$ \\ Yutaka Fujimoto, ${ }^{1}$ and Keisuke Asai ${ }^{1}$ \\ ${ }^{1}$ Department of Applied Chemistry, Graduate School of Engineering, Tohoku University, \\ 6-6-07 Aoba, Aramaki, Aoba-ku, Sendai 980-8579, Japan \\ ${ }^{2}$ Division of Materials Science, Nara Institute of Science and Technology, \\ 8916-5 Takayama-Cho, Ikoma, Nara 630-0192, Japan \\ ${ }^{3}$ Plaken Co. Ltd., 168-8 Tsurugi, Kashiwazaki, Niigata 945-0105, Japan
}

(Received November 29, 2018; accepted March 25, 2019)

Keywords: plastic scintillator, $\alpha$-ray, thin film

The photoluminescence and scintillation properties of thin-film plastic scintillators of 5-100 $\mu \mathrm{m}$ thicknesses have been analyzed. The photoluminescence and X-ray-induced radioluminescence spectra exhibited a peak at $400-450 \mathrm{~nm}$, which is typical for generic plastic scintillators. As for the radioluminescence spectra, the contribution of the short-wavelength component decreased with the increase in thickness owing to the self-absorption of scintillation photons inside the scintillators themselves. The temporal profiles of scintillation exhibited a fast decay on the order of nanoseconds. The pulse-height spectra of the scintillation detectors equipped with thin-film plastic scintillators were obtained for $5.4 \mathrm{MeV} \alpha$-rays from ${ }^{241} \mathrm{Am}$ to evaluate the $\alpha$-ray detection capabilities. Peaks were observed for thin-film plastic scintillators of 9 and $22 \mu \mathrm{m}$ thicknesses, and their light yields were estimated to be 830 and 410 photons $/ \mathrm{MeV}$, respectively. The dependence of pulse height spectra on film thickness indicates that selfabsorption occurs in such thin films.

\section{Introduction}

Plastic scintillators have long been used for various applications. One of the advantages of plastic scintillators is their fast response: the scintillation decay-time constant of generic plastic scintillators is less than $10 \mathrm{~ns}$. The plastic scintillators have been used in applications in which good time resolution or capability of detection at a high counting rate is necessary. Another advantage is the relative ease of fabrication and processing. In contrast to inorganic singlecrystal scintillators, plastic scintillators can be easily fabricated on a large scale. In addition, they can be easily processed into complicated shapes. This advantage is further strengthened by the use of 3D printers. ${ }^{(1)}$ Furthermore, they can be fabricated into thin films.

Presently, plastic scintillation thin films of less than $100 \mu \mathrm{m}$ thickness are produced by Plaken Co., Ltd. and are available at Fujitok Corporation. A possible application of such thin

*Corresponding author: e-mail: koshi@qpc.che.tohoku.ac.jp

https://doi.org/10.18494/SAM.2019.2182 
films is the detection of $\alpha$-rays. Because the range of $\alpha$-rays in solids is generally up to several tens of microns, thin films are suitable for the detection of $\alpha$-rays. The low atomic numbers of the constituent elements $(\mathrm{C}, \mathrm{H}, \mathrm{O}$, and so forth) lead to a low probability of interaction with high-energy photons, and hence high-energy charged particles such as $\alpha$-rays can be detected with a low $\gamma$-ray background. In addition, considering the basic properties of plastic scintillators, it is appropriate to use thin films to analyze the thickness-dependent scintillation properties, including the self-absorption of scintillation photons inside the plastic scintillators themselves, which is a common problem occurring in plastic scintillators. ${ }^{(2)}$

In this study, the photoluminescence and scintillation properties of thin-film plastic scintillators of different thicknesses in the range of 5-100 $\mu \mathrm{m}$ were analyzed. As for the scintillation properties, X-ray-induced radioluminescence and temporal profiles of scintillation were measured. In addition, to evaluate the $\alpha$-ray detection capabilities, the pulse height spectra of the scintillation detectors equipped with the thin-film plastic scintillators were obtained for $5.4 \mathrm{MeV} \alpha$-rays from ${ }^{241} \mathrm{Am}$. The dependence of scintillation properties on film thickness was analyzed from the viewpoint of the self-absorption of scintillation photons inside the scintillators themselves.

\section{Experimental Procedure}

Plastic scintillator films based on polyvinyltoluene were supplied by Plaken Co., Ltd. The thicknesses of the films were $5,9,22,50,61,78$, and $100 \mu \mathrm{m}$. The composition of the films was equivalent to that of $\mathrm{BC}-400$. The photoluminescence spectra were measured using a spectrofluorometer (F-7000, Hitachi). X-ray-induced radioluminescence spectra were measured using an X-ray generator (XRB80N100/CB, Spellman) operating at $40 \mathrm{kV}$ and $1.2 \mathrm{~mA}$. The samples were attached to an optical fiber and irradiated with X-rays. The scintillation from the samples was delivered with an optical fiber to a CCD detector (DU-920-P, Andor) equipped with a monochromator (SR163i-UV, Andor). ${ }^{(3)}$ The temporal profiles of scintillation were obtained using a pulsed X-ray beam as the excitation source and a time-correlated single-photon counting technique. ${ }^{(4)}$ The scintillation from the samples was detected with a photomultiplier tube (PMT; R7400P-06). The pulse-height spectra were obtained for $5.4 \mathrm{MeV} \alpha$-rays in the following manner. The scintillator films were attached to another PMT (R877-100, Hamamatsu Photonics) with an optical grease. The scintillators were irradiated with $\alpha$-rays from an ${ }^{241} \mathrm{Am}$ source placed on the scintillators. The detection signals of the PMT were fed to a preamplifier (113, Ortec) and subsequently to a shaping amplifier (572A, Ortec). Finally, the pulse-height spectra were obtained with a multichannel analyzer (8000A, Amptek).

\section{Results and Discussion}

Figure 1 shows the photoluminescence spectra with excitation at $200 \mathrm{~nm}$, which corresponds to the photon energy well above the difference in HOMO-LUMO energy. For the films of 61, 78 , and $100 \mu \mathrm{m}$ thicknesses, a dominant peak was observed at approximately $420 \mathrm{~nm}$, which is consistent with the data sheet of BC-400. For the thinner films, the contribution on the short- 

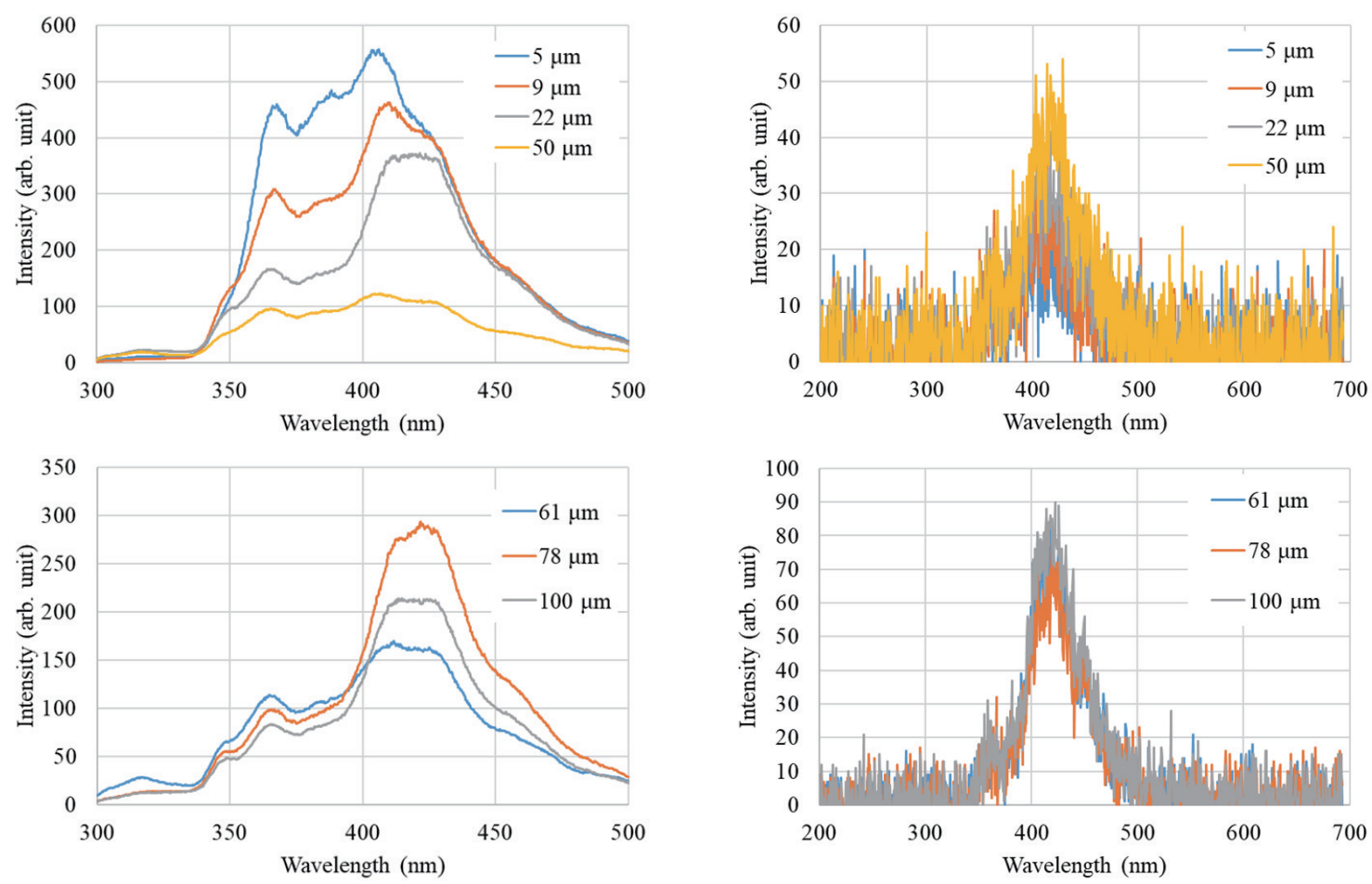

Fig. 1. (Color online) Photoluminescence spectra of thin-film plastic scintillators. Excitation wavelength: $200 \mathrm{~nm}$.

Fig. 2. (Color online) X-ray-induced radioluminescence spectra.

wavelength side of the spectrum was greater. The results suggest that the radiative energy transfer, i.e., the excitation of the secondary fluorescence compounds upon the absorption of photons emitted by the primary fluorescence compounds, is not effective owing to the low probability of the absorption of the photons emitted by the primary fluorescence compounds. The film thicker than $50 \mu \mathrm{m}$ exhibited similar spectra, which suggests that the contribution of the radiative energy transfer saturates over $50 \mu \mathrm{m}$.

Figure 2 shows the X-ray-induced radioluminescence spectra. The signal-to-noise ratio is low owing to the low X-ray stopping power of the thin-film plastic scintillators. The emission ranged over $350-600 \mathrm{~nm}$ with peaks at $400-500 \mathrm{~nm}$ for all the scintillator films, which is consistent with the photoluminescence spectra. The contribution of the longwavelength component is more prominent for the radioluminescence spectra than for the photoluminescence spectra, which is possibly owing to the difference in measurement geometry: the photoluminescence and radioluminescence spectra were measured in reflection and transmission geometries, respectively. The self-absorption of the emitted photons in the films themselves is more significant in transmission geometry, which results in the reduction in the contribution of the short-wavelength component.

Figure 3 shows the temporal profiles of the scintillation of the thin-film plastic scintillators. The temporal profiles were superimposed with fitted curves representing the sum of two exponential decay functions and a time-independent component. The decay-time constants of 

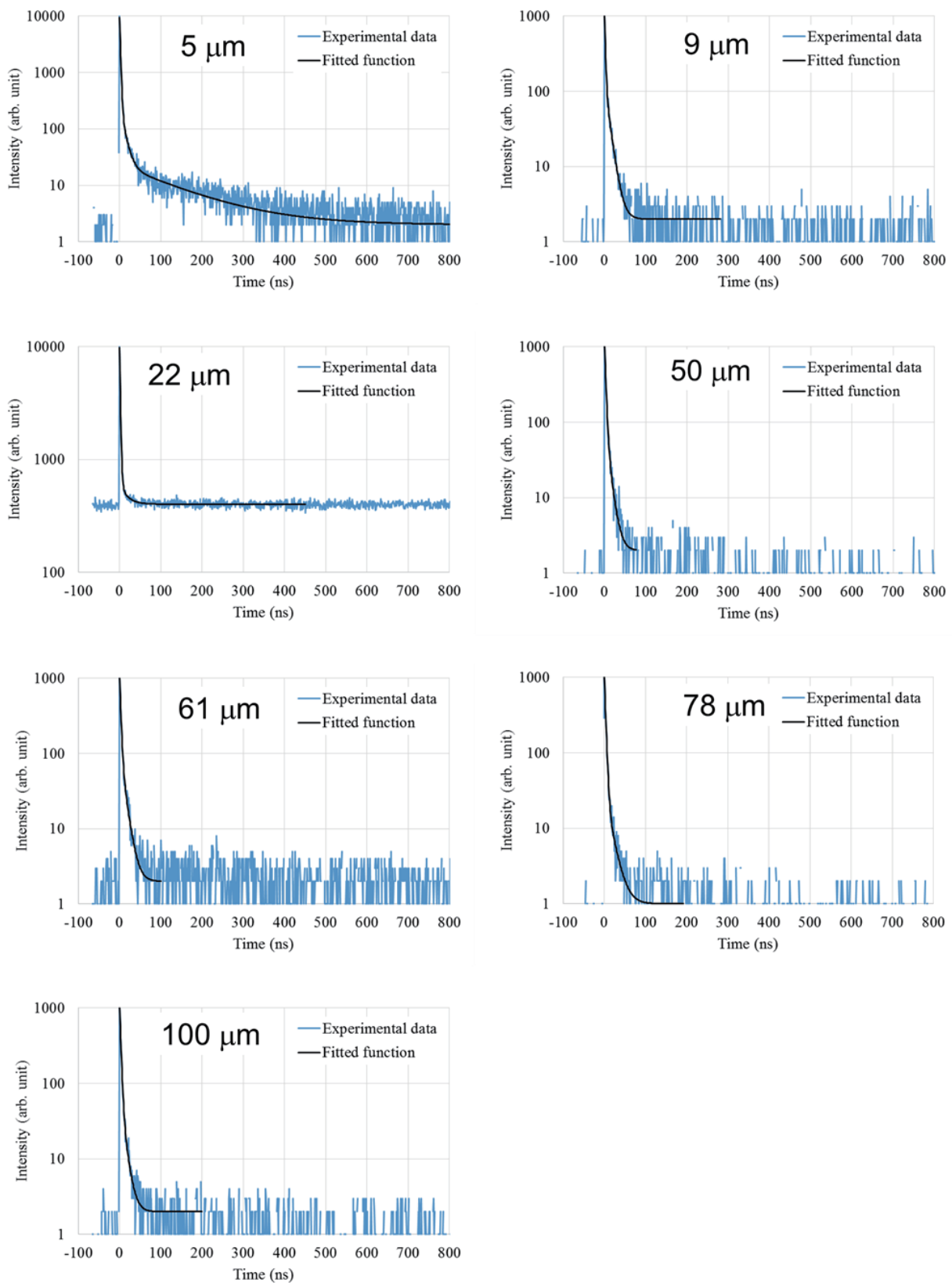

Fig. 3. (Color online) Temporal profiles of scintillation of thin-film plastic scintillators. Fitted functions represent the sum of two exponential decay functions and a time-independent component.

the decay components and their relative intensities are summarized in Table 1. The decay-time constant of the 1st component was approximately $2 \mathrm{~ns}$, which is typical for plastic scintillators. The decay-time constants of the plastic scintillators of 5 and $9 \mu \mathrm{m}$ thicknesses were significantly smaller than those of the thicker scintillators, which suggests that the emission of the primary fluorescence compounds was dominant in the thin-film scintillators. In addition to the 1 st component, the 2 nd component was also observed, whose decay-time constant was 
Table 1

Decay-time constants and relative intensities (in parentheses) of decay components in temporal profiles of scintillation.

\begin{tabular}{ccr}
\hline Thickness $(\mu \mathrm{m})$ & 1st component & 2nd component \\
\hline 5 & $1.8 \mathrm{~ns}(55 \%)$ & $9.6 \mathrm{~ns}(45 \%)$ \\
9 & $1.9 \mathrm{~ns}(66 \%)$ & $10.5 \mathrm{~ns}(34 \%)$ \\
22 & $2.7 \mathrm{~ns}(74 \%)$ & $13.4 \mathrm{~ns}(26 \%)$ \\
50 & $2.6 \mathrm{~ns}(80 \%)$ & $9.8 \mathrm{~ns}(20 \%)$ \\
61 & $2.6 \mathrm{~ns}(75 \%)$ & $10.8 \mathrm{~ns}(25 \%)$ \\
78 & $2.7 \mathrm{~ns}(89 \%)$ & $14.9 \mathrm{~ns}(11 \%)$ \\
100 & $2.7 \mathrm{~ns}(84 \%)$ & $10.1 \mathrm{~ns}(16 \%)$ \\
\hline
\end{tabular}

Table 2

Average deposited energy of $5.4 \mathrm{MeV} \alpha$-rays in thinfilm plastic scintillators of different thicknesses estimated with SRIM code.

\begin{tabular}{cc}
\hline Thickness $(\mu \mathrm{m})$ & Deposited energy $(\mathrm{MeV})$ \\
\hline 5 & 0.47 \\
9 & 0.87 \\
22 & 2.5 \\
Over 40 & 5.4 \\
\hline
\end{tabular}

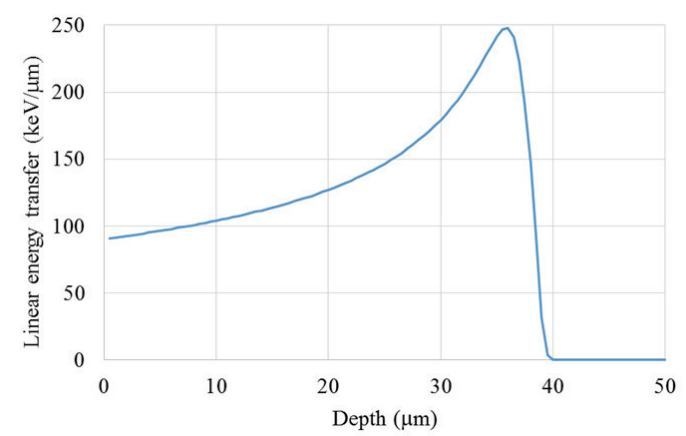

Fig. 4. (Color online) Linear energy transfer estimated for $5.4 \mathrm{MeV} \alpha$-rays from 241Am with SRIM code.

approximately $10 \mathrm{~ns}$. The contribution of the 2 nd component was greater for the thinner films, which suggests that the 2nd component may be attributed to the excited states near the surface.

Figure 4 shows the linear energy transfer, i.e., the deposited energy per unit length of the trajectory, of $5.4 \mathrm{MeV} \alpha$-rays from ${ }^{241} \mathrm{Am}$ in the plastic scintillator, BC-400, with the Stopping and Range of Ions in Matter (SRIM) code. ${ }^{(5)}$ The angle of injection to the scintillator was set at $90^{\circ}$. The $\alpha$-rays are estimated to deposit their entire energy and stop within $40 \mu \mathrm{m}$ from the surface. Table 2 shows the average deposited energy of $5.4 \mathrm{MeV} \alpha$-rays in the thin-film plastic scintillators of different thicknesses estimated with the SRIM code. On the basis of this estimation, the entire energy of the $\alpha$-rays is deposited within the films thicker than $50 \mu \mathrm{m}$ in this study.

Figure 5 shows the pulse-height spectra of the scintillation detectors equipped with the thin-film plastic scintillators for $5.4 \mathrm{MeV} \alpha$-rays from ${ }^{241} \mathrm{Am}$. The pulse-height spectra of a scintillation detector equipped with $\mathrm{Ce}: \mathrm{Gd}_{2} \mathrm{SiO}_{5}$ (GSO) for $662 \mathrm{keV} \gamma$-rays from ${ }^{137} \mathrm{Cs}$ are shown for comparison. For the thin films of $5 \mu \mathrm{m}$ thickness, a shoulder was observed at approximately 170 channels. For the thin films of 9 and $22 \mu \mathrm{m}$ thicknesses, clear peaks were observed at 140 and 190 channels, respectively. The scintillation light yields for these films can be estimated as follows. The photoelectron peak is observed for GSO, whose light yield was estimated to be 10000 photons $/ \mathrm{MeV}$, at approximately 320 channel, which results in 6620 photons corresponding to 320 channels. The coarse gains for the measurements of GSO and the thin-film scintillators 

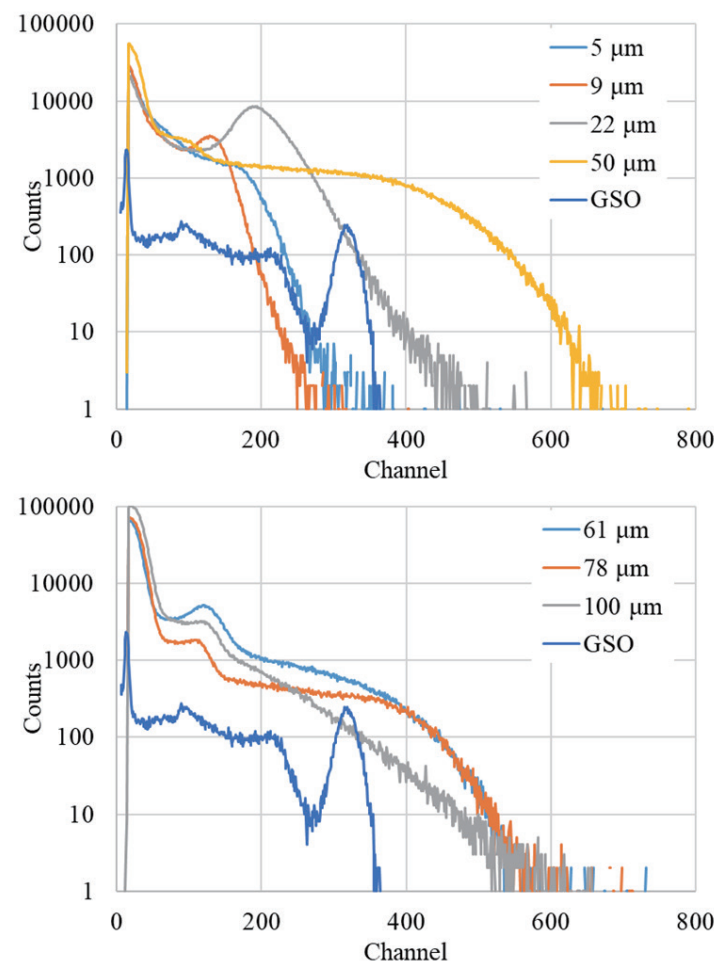

Fig. 5. (Color online) Pulse-height spectra of scintillation detectors equipped with thin-film plastic scintillators for 5.4 MeV $\alpha$-rays from ${ }^{241} \mathrm{Am}$. Pulse-height spectra of a scintillation detector equipped with GSO for $662 \mathrm{keV} \gamma$-rays from ${ }^{137} \mathrm{Cs}$ are shown for comparison. The coarse gains for the measurements of GSO and thin-film scintillators were 50 and 200 , respectively.

were 50 and 200, respectively. From these results, the clear peaks for the yields of thin films of 9 and $22 \mu \mathrm{m}$ thicknesses correspond to 720 and 980 photons, respectively. From the estimation with the SRIM code, the average deposited energies of $5.4 \mathrm{MeV} \alpha$-rays in these films are 0.87 and $2.5 \mathrm{MeV}$, respectively. Hence, the light yields for these films are 830 and 410 photons/ $\mathrm{MeV}$, respectively. These values are reasonable considering that the light yield exceeds 10,000 photons $/ \mathrm{MeV}\left[65 \%\right.$ anthracene for $\mathrm{BC}-400^{(6)}$ and that the light yield of anthracene for $\alpha$-rays is 20000 photons/MeV estimated recently with measurements of the Compton edge, ${ }^{(7)}$ or 17400 photons $/ \mathrm{MeV}$ deduced from the light yields for various scintillators $\left.{ }^{(8)}\right]$ and considering that the $\mathrm{a} / \mathrm{b}$ ratio is less than 0.1 for generic plastic scintillators. ${ }^{(9)}$

As for the dependence on thickness, the pulse height spectra extended toward the largechannel side up to $50 \mu \mathrm{m}$. The extension of the spectra indicates an increase in the number of scintillation photons. This trend is due to the increased deposited energy within the thinfilm scintillators up to $50 \mu \mathrm{m}$ as estimated with the SRIM code. The other trend is the shift toward the small-channel side for the films thicker than $50 \mu \mathrm{m}$. The high channel edge and the shoulder of the spectra shifted toward the small-channel side with the thickness in the pulse height spectra. A plausible cause for this shift is the self-absorption of the scintillation photons inside the scintillators themselves. The lower light yield of the $22 \mu \mathrm{m}$ film of 410 photons/MeV as compared with the $9 \mu \mathrm{m}$ film of 830 photons/ $\mathrm{MeV}$ is also explained by self- 
absorption. Furthermore, this is in line with the difference between the photoluminescence and radioluminescence spectra. In the radioluminescence spectra, which were obtained in transmission geometry, the contribution of the short-wavlenegth component is lower than that of the photoluminescence spectra that were obtained in reflection geometry.

\section{Conclusions}

The photoluminescence and scintillation properties of thin-film plastic scintillators of 5-100 $\mu \mathrm{m}$ thicknesses have been analyzed. The photoluminescence and radioluminescence spectra obtained were typical for generic plastic scintillators, although the contribution of the shortwavelength component in the radioluminescence spectra was low for thick films owing to the self-absorption of the emitted photons inside the thin films themselves. The thin-film plastic scintillators exhibited fast scintillation decay, which is typical for generic plastic scintillators. The pulse height spectra for $5.4 \mathrm{MeV} \alpha$-rays from ${ }^{241} \mathrm{Am}$ exhibited clear peaks for the thin films of 9 and $22 \mu \mathrm{m}$ thicknesses. By comparing the peak position to that for GSO and the deposited energy estimated with the SRIM code, the light yields for the thin films of 9 and 22 $\mu \mathrm{m}$ thicknesses are estimated to be 830 and 410 photons/MeV, respectively. The pulse height spectra were also influenced by the self-absorption of the scintillation photons inside the thin films themselves.

\section{Acknowledgments}

This research was supported by a Grant-in-Aid for Scientific Research (A) (No. 18H03890, 2018-2021). Part of this research is based on the Cooperative Research Project of the Research Center for Biomedical Engineering, Ministry of Education, Culture, Sports, Science and Technology.

\section{References}

1 Y. Mishnayot, M. Layani, I. Cooperstein, S. Magdassi, and G. Ron: Rev. Sci. Instrum. 85 (2014) 08510.

2 V. Senchyshyn, V. Lebedev, A. Adadurov, J. Budagov, and I. Chirikov-Zorin: Nucl. Instrum. Methods Phys. Res., Sect. A 566 (2006) 286.

3 T. Yanagida, K. Kamada, Y. Fujimoto, H. Yagi, and T. Yanagitani: Opt. Mater. 35 (2013) 2480.

4 T. Yanagida, Y. Fujimoto, T. Ito, K. Uchiyama, and K. Mori: Appl. Phys. Express 7 (2014) 062401.

5 J. F. Ziegler, J. P. Biersack, and U. Littmark: The Stopping and Range of Ions in Solids (Pergamon Press, New York, 1984).

6 Datasheet of BC-400, BC-404, BC-408, BC-412, BC-416: https://www.crystals.saint-gobain.com/sites/imdf. crystals.com/files/documents/bc400-404-408-412-416-data-sheet.pdf

7 T. Yanagida, K. Watanabe, and Y. Fujimoto: Nucl. Instrum. Methods Phys. Res., Sect. A 784 (2015) 111.

8 G. F. Knoll: Radiation Detection and Measurements (Wiley, New York, 2000).

9 W. Wolszczak and P. Dorenbos: IEEE Trans. Nucl. Sci. 64 (2017) 1580. 